\title{
DOCUMENTOS
}

\section{Acciones de Achalai para la recuperación del patrimonio sonoro musical prehispánico}

\author{
por \\ Lina Barrientos \\ Universidad de La Serena \\ lbarrien@userena.cl \\ José Pérez de Arce \\ Museo Chileno de Arte Precolombino \\ jperezdearce@museoprecolombino.cl
}

\section{INTRODUCGIÓN}

A mediados del año 2007 un grupo de cinco investigadores italianos, entre ellos el compositor y pianista del Conservatorio de Parma, Francesco de Mattia, quien actúa como coordinador artístico del grupo, dieron a conocer los primeros resultados de lo que fuera el Proyecto ASTRA, Ancient Instrument Sound / Timbre Reconstruction Application. Este proyecto se centró en la reconstrucción de sonidos de instrumentos musicales antiguos. La información ha sido obtenida mediante datos arqueológicos, provenientes de restos fragmentados y/o dibujos y pinturas. La reconstrucción sonora es realizada por medio del modelamiento físico-matemático, basado en el uso de un set de ecuaciones y algoritmos que describen el material físico usado en el instrumento para generar las condiciones físicas del sonido (ASTRA, website).

Uno de los primeros instrumentos sometidos a este proceso de modelamiento fue el epigonion de la Antigua Grecia. Se logró reconstituir los sonidos de sus 48 cuerdas, los que fueron almacenados en un medio magnético, para ser reproducidos por un sitema MIDI o ejecutados en un teclado convencional (Reyes, 2011). Los sonidos reconstituidos de este epigonion fueron parte de un concierto por primera vez el 4 de noviembre de 2008. La interpretación estuvo a cargo de Francesco de Mattia, quien junto a otros músicos conforma la Lost Sound Orchestra, cuyo propósito es difundir la música interpretada con sonidos reconstituidos de instrumentos antiguos.

Dentro de este espíritu de búsqueda de sonoridades producidas por instrumentos antiguos, Francesco de Mattia amplió el área geográfica hacia América Latina. Para ello tomó contacto con RedCLARA, Red de Cooperación Latinoamericana de Redes Avanzadas, la que opera en el medio académico y mediante REUNA, Red Universitaria Nacional de Chile ${ }^{1}$, conformó un equipo de investigadores de áreas ligadas con la musicología, física

1 REUNA es la red chilena de RedCLARA.

Revista Musical Chilena, Año LXVII, enero-junio, 2013, No 219, pp. 81-89 
acústica e ingeniería informática procedentes de ocho países iberoamericanos. Así surgió la Comunidad Achalai. Su propósito es recuperar y revalorizar un patrimonio cultural e inmaterial de tradiciones musicales prehispánicas a partir del modelamiento físico de instrumentos antiguos para la recuperación de sus sonidos. Para ello se utilizan tecnologías de información avanzadas capaces de posibilitar esta restauración. Así se ha obtenido una información valiosa para el estudio de las culturas musicales del pasado prehispánico de América, lo que ha permitido la reinterpretación de los sonidos musicales recuperados por parte de la comunidad musical y artística.

Para la ejecución del proyecto se contó con el apoyo de dos instituciones que operan y administran fondos de la Comunidad Europea. Una de ellas es CYTED², Programa Iberoamericano de Ciencia y Tecnología para el Desarrollo, Área Temática Ciencia y Sociedad, mediante la Línea de Investigación Patrimonio Cultural y Multiculturalidad. Este programa financia la movilidad de los investigadores para reuniones de organización, evaluación y estudio de temáticas específicas, como también para la difusión de los resultados. La otra es la RedCLARA ${ }^{3}$, anteriormente mencionada, la que aporta internet avanzado para la conectividad operativa de la red de académicos como también e-infraestructura para el almacenamiento de información, específicamente en lo relativo al modelamiento físico de los instrumentos.

\section{COMUNIDAD ACHALAI}

La comunidad Achalai estaba conformada el año 2012 por 29 investigadores iberoamericanos de diversas disciplinas, quienes de alguna manera están relacionados en lo musical. A modo de ejemplo se cuenta con un físico belga-boliviano que toca aerófonos y se integra a tropas de siku y tarkas de comunidades rurales autóctonas; un ingeniero mexicano que se la ha jugado a nivel gubernamental por el reconocimiento de la importancia que tiene el rescate de sonoridades producidas por objetos sonoros y/o instrumentos musicales prehispánicos para la vida actual, especialmente la salud; un ingeniero eléctrico chileno y flautista con un doctorado en computación basado en estudios de teoría musical y acústica. A ellos se suman sociólogos, arqueólogos, musicólogos, un museólogo, una diseñadora y una ceramista, entre otros, además de músicos intérpretes y compositores.

Durante una reunión presencial realizada en Santiago de Chile en julio del año 2011, se establecieron comisiones de trabajo por especialidad. Se conformaron cuatro comisiones, una encargada de la gestión del proyecto, otra para la difusión y dos comisiones medulares: la Comisión de Modelamiento y la Comisión de Acústica/Instrumentos Prehispánicos, encargada de la preselección y estudio de los instrumentos que finalmente serán seleccionados por su factibilidad, para el modelamiento físico. Los integrantes de la Comisión Acústica/Instrumentos Prehispánicos preseleccionamos un corpus de instrumentos para estudiar. Se fundamentó que, por las características predominantes de los objetos sonoros y/o instrumentos musicales conservados en museos estos debían ser aerófonos, por ser el sonido que ha prevalecido en el tiempo y que ha identificado a América Latina. Una segunda condición que se consideró para la selección de los instrumentos fue que

2 Proyecto Achalai/CYTED, Red de acción colaborativa de recuperación musical prehispánica ancestral, armonizando investigación y tecnología. Francesco de Mattia, coordinador en una primera etapa. Málaga, España: Universidad de Málaga. Desde el segundo semestre de 2012 asumió la coordinación Paulina López, Subgerente de proyectos de REUNA-Chile.

3 Proyecto Achalai/ComCLARA, Red internacional de recuperación del patrimonio inmaterial de tradiciones musicales. Lina Barrientos, organizadora principal. La Serena, Chile: Universidad de La Serena. 
se contara con el proceso de modelamiento físico al cual se someterían para reconstituir sus sonidos. Por lo tanto se debía aprovechar la instancia para trabajar con instrumentos que estuvieran en muy mal estado, rotos o erosionados y que además fueran particulares y representativos de una cultura ya extinguida. Después de una exposición fotográfica de varios instrumentos, presentada por tres investigadores, que incluían descripciones de sus condiciones, antecedentes culturales y datos arqueológicos de sus hallazgos, se seleccionó en primera instancia a cinco instrumentos, uno de cada país presente en la Comunidad Achalai, a los que se integró posteriormente un sexto.

\begin{tabular}{|c|c|c|c|}
\hline País & Instrumento & Investigador a cargo & Institución \\
\hline Ecuador & botella silbadora & Mónica Polanco & $\begin{array}{c}\text { Pontificia Universidad } \\
\text { Católica de Ecuador } \\
\text { sede Ibarra }\end{array}$ \\
\hline Perú & antara Paracas & Carlos Mansilla & $\begin{array}{c}\text { Escuela Nacional Superior } \\
\text { del Folclor } \\
\text { José María Arguedas }\end{array}$ \\
\hline Chile & pito acodado & José Pérez de Arce & $\begin{array}{l}\text { Museo Chileno de Arte } \\
\text { Precolombino }\end{array}$ \\
\hline Bolivia & ayarachi Yura & Arnaud Gérard & $\begin{array}{l}\text { Universidad Mayor de } \\
\text { San Andrés, La Paz }\end{array}$ \\
\hline Colombia & ocarina Zenú & Álvaro Bermejo & Universidad del Atlántico \\
\hline México & ilmenita sonora Olmeca & Roberto Velázquez & $\begin{array}{l}\text { Instituto Virtual } \\
\text { de Investigación } \\
\text { Tlapitzcalzin }\end{array}$ \\
\hline
\end{tabular}

La modalidad de trabajo ha sido que cada investigador a cargo, en sus respectivos países, conforme un equipo de trabajo interinstitucional e interdisciplinario, con el propósito de profundizar en el estudio del objeto sonoro o instrumento musical bajo su responsabilidad. En un principio nos nutrimos con una bibliografía específica producida por los propios miembros de Comunidad Achalai en las que exponen resultados de sus investigaciones, hipótesis y reflexiones. Estas publicaciones fueron pasadas a formato digital y subidas a la wiki del portal de RedCLARA y a la página web de Achalai, a la que tuvieron acceso todos los miembros. Utilizando internet avanzado, se realizaron reuniones de trabajo virtuales en tiempo real mediante videoconferencias, para lo que se utilizó el huso horario GMT como medio de coordinación. La conexión se efectuó con las redes académicas de América Latina. Paralelamente se utilizó la modalidad streaming, mediante la cual es posible seguir la reunión solo con conexión a internet, en el caso de aquellos investigadores de Francia y España o de aquellos que eventualmente no tienen acceso a la Red y que pudieron participar en las reuniones utilizando skype.

Gracias a esta modalidad de videoconferencia se han efectuado cuatro clase-conferencias virtuales dirigidas al mundo académico de las propias instituciones a las que pertenecen 
los miembros de la Comunidad Achalai. Estas clase-conferencias se efectuaron en octubre y noviembre de 2011 y abril y mayo de 2012. En la primera participaron investigadores de cinco países: Chile, Bolivia, Colombia, Ecuador y Perú. Se abocó a la presentación de cinco instrumentos prehispánicos en estudio. En la segunda participaron investigadores de tres países (Colombia, Bolivia y Chile). Se abocó a los procedimientos físico-acústicos para el estudio de los instrumentos, los que son fundamentales para suministrar los antecedentes que permitan la elección del instrumento más adecuado o de características más interesantes para la modelación física. Las otras dos clase-conferencias estuvieron dedicadas específicamente a dos objetos sonoros que forman parte del corpus en estudio: la botella silbadora de Ecuador, por su belleza y gran variedad de formas encontradas y la ilmenita sonora olmeca de México, por su extrañeza, por el gran número que se ha encontrado y por la incógnita de su funcionalidad y/o cómo fue utilizada.

El proyecto considera tres ejes: el primero relacionado con la selección y estudio de seis objetos sonoros o instrumentos musicales prehispánicos; el segundo relacionado con la reconstitución del sonido de uno o dos de esos objetos o instrumentos por medio de la modelación físico-matemática, y el tercero relacionado con la realización de un concierto en el que una obra musical sea interpretada con el o los sonidos recuperados. Respecto de este último eje, apareció una reflexión ética por parte del grupo de musicólogos, etnomusicólogos y arqueomusicólogos. Se invitó a examinar las culturas en que estos instrumentos fueron utilizados. Por los conocimientos de que disponemos, la música, o lo que nosotros distinguimos como música, para las culturas originarias ha tenido y tiene una connotación ligada a una funcionalidad ceremonial-ritual, y en la mayoría de las ocasiones la ejecución de un instrumento musical está relacionado con sistemas de creencias y explicaciones cosmogónicas.

Es así como concordamos en entregar, junto a los procedimientos metodológicos para el estudio del instrumento, un manual de interpretación, un protocolo de uso de los sonidos reconstituidos en el marco del Proyecto Achalai y un manual de ejecución que contenga referentes étnicos del uso del instrumento. Respecto de la ejecución musical de los instrumentos, nos pareció interesante proponer una fórmula paralela, distinta a la de Lost Sound Orchestra, para recoger variables propias de América en la ejecución musical. De este modo habrá dos canales para la expresión musical que conocerá el público: una es la de Lost Sound Orchestra, basada en cánones musicales europeos, y la otra, que se generará durante el proyecto, basada en cánones musicales americanos.

\section{SEIS INSTRUMENTOS PREHISPÁNICOS}

La elección de los instrumentos siguió dos parámetros principales. Uno de ellos es la representación geográfica, de modo que los distintos países participantes pudieran estar representados por un ejemplar. El otro criterio es organológico. Supone tener una muestra que contemple distintas familias de instrumentos y ojalá especies organológicas vernáculas endémicas, que no estén representadas en otras zonas del planeta y que por lo tanto representen situaciones inéditas respecto del objeto de estudio. Existe claridad en cuanto a que este planteamiento es sumamente preliminar. Servirá no para presentar un panorama de la organología prehispánica de la región, sino que para intentar un primer paso en el conocimiento de una zona muy restringida, pero altamente interesante de este panorama.

A continuación se presenta una breve descripción de los objetos elegidos. 


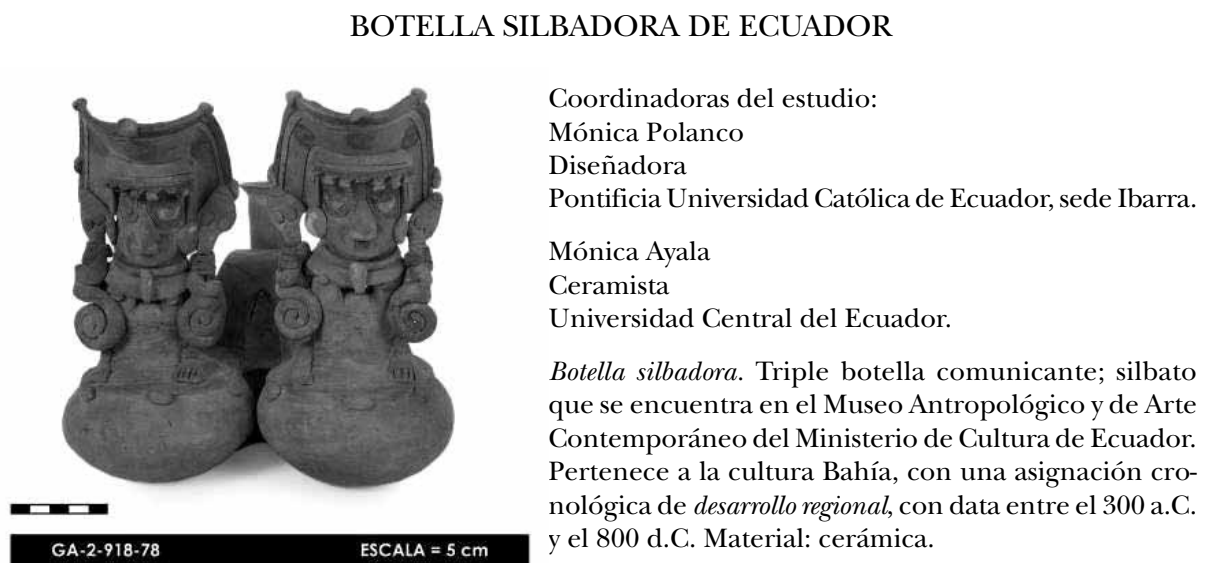

La botella silbadora, de amplia distribución en los Andes y Mesoamérica, tuvo sus inicios en la cultura Chorrera de Ecuador (1200-200 a.C.), en la que se alcanzó un alto grado de perfección tanto artesanal visual como acústica. La acústica de estos objetos está íntimamente relacionada con el líquido que contienen. Este líquido puede provocar el sonido sin intervención del soplo humano, en cuyo caso los sonidos provocan interesantes y poco estudiados fenómenos acústicos y respuestas sonoras. Luego de su enorme difusión espacial, este tipo de objetos desaparece después de la conquista europea.

\section{ANTARA PARACAS DE PERÚ}

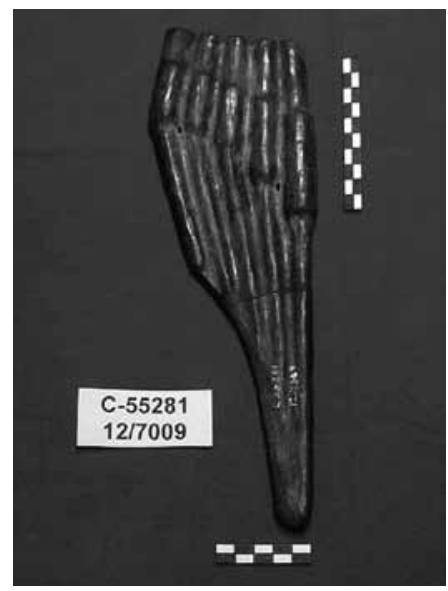

Coordinadores del estudio:

Carlos Mansilla

Musicólogo

Escuela Nacional Superior del Folclor José María Arguedas, Perú.

Milano Trejo

Investigador organológico.

Museo Nacional de Arqueología, Antropología e Historia del Perú.

Antara Paracas. Pertenece a la cultura Paracas o Wari Kayan, de la época del 200 a.C. hasta el 150 d.C., ubicada en el Distrito Paracas, Provincia Nasca, Departamento Ica.

Es un instrumento musical aerófono encontrado por Julio Tello en 1927.

Es de cerámica de tubos complejos, por tener tres dimensiones cada uno de ellos, su largo es de $28,5 \mathrm{~cm}$.

La antara, nombre aymara de una especie de flauta de Pan, se refiere en el proyecto a un tipo especial de flauta de Pan que existió en una amplia zona de los Andes Sur, entre el sur de Perú y la Región de Los Lagos en Chile durante más de 2000 años, y que se extinguió luego de la conquista europea. El origen de toda esta gran familia instrumental está en la cultura Paracas (700 a.C.-200 d.C.), de la costa sur de Perú, en la que se dan ejemplares excepcionales por sus características organológicas, sin que se sepa a ciencia cierta a qué corresponden esas características en un sentido musical o sonoro. 


\section{PITO ACODADO DE CHILE}

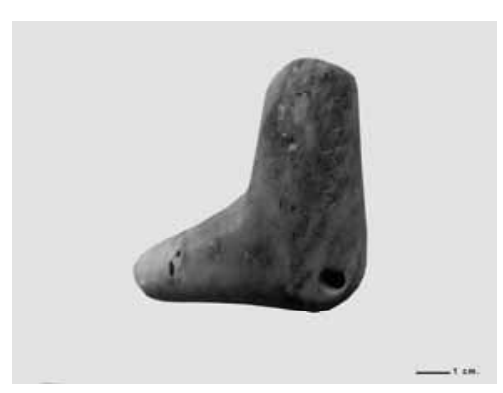

Coordinadores del estudio:

José Pérez de Arce

Museógrafo, arqueomusicólogo, músico

Museo Chileno de Arte Precolombino, Chile.

Francisca Gili

Conservadora. Estudiante de postgrado en Arqueología Universidad Católica del Norte, Chile.

Flauta acodada. La pieza fue recuperada de un proyecto de impacto ambiental en el sector El Olivar de la Compañía Baja de La Serena, el año 2010, por los arqueólogos Gabriel Cantarutti y Gloria Cabello. En la actualidad se encuentra en calidad de préstamo en el Museo Chileno de Arte Precolombino para su estudio. Después será depositada en el Museo Arqueológico de La Serena. Se cree que pertenece a la cultura Diaguita, del período intermedio tardío, con data entre 1100 y 1250 d.C. Es de material lítico, de un solo tubo en forma acodada, con diámetro de dos dimensiones, como las flautas de tubo complejo. Su tamaño es de $13 \times 2,5 \mathrm{~cm}$, aproximadamente.

El hallazgo de varias flautas con una característica en forma de "L", de la que se ha tomado su nombre, en la región de Norte Chico y Valles Centrales de Chile plantea varias incógnitas que están sin resolver. El objeto escogido para este proyecto, del Museo de La Serena, corresponde a la cultura diaguita (1100-1250 d.C.), es muy característico de esta especie organológica y está en condiciones de ser estudiado. Por una parte su forma plantea una interesante relación con otras familias de flautas, junto con establecer vínculos con pipas para fumar, por la otra. Esta ambigüedad, que se encuentra también en la región de La Araucanía, usos ceremoniales chamánicos, y al mismo tiempo establece una relación con sonidos propios de los rituales regionales, el llamado "sonido rajado", con relación a estructuras acústicas inéditas cuya influencia en el sonido resultante no es conocida.

\section{AYARACHI YURA DE BOLIVIA}

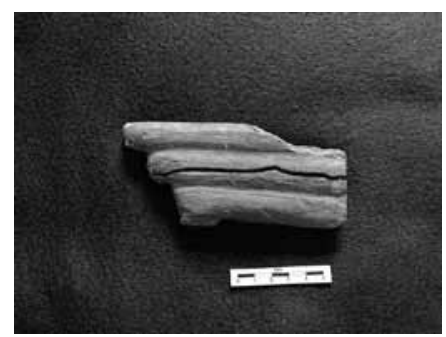

Coordinadores del estudio:

Arnaud Gérard

Físico acústico, músico

Instituto de Investigaciones Físicas

Universidad Mayor de San Andrés, La Paz, Bolivia.

Edmundo Salinas

Arqueólogo

Museo Charcas de Sucre

Instituto de Investigación Antropológica y Arqueológica

Universidad de San Francisco Xavier de Chuquisaca, Sucre, Bolivia.

Ayarachi Yura. Pertenece a la cultura Yura ubicada en Potosí, del período “intermedio tardío”, entre el 900 y el 1400 d.C.

Es una flauta de Pan lítica de 13 x 5,5 cm con tres tubos complejos de dos dimensiones.

El instrumento fue extraído junto a un lote de 56 objetos en el año 1956.

Se trata de una flauta de Pan lítica de la cultura Yura (900-1400 d.C.) de tres "tubos complejos" del Museo Charcas de Sucre - Instituto de Investigación Antropológica y Arqueológica - Universidad Mayor Real y Pontífice San Francisco Xavier de Chuquisaca-Sucre en Bolivia, que por su actual estado no puede ser tañida, pero de la cual se espera poder reconstruir el sonido. Este sonido debería corresponder al "sonido rajado" que producen otros instrumentos de la región con el mismo tipo de tubo sonoro. El interés de este instrumento es que proviene de una región que probablemente está relacionada con el inicio de las llamadas "antaras surandinas", que se extendieron hasta el sur de Chile y que desaparecieron con la llegada del conquistador europeo. 


\section{OCARINA ZENÚ DE COLOMBIA}

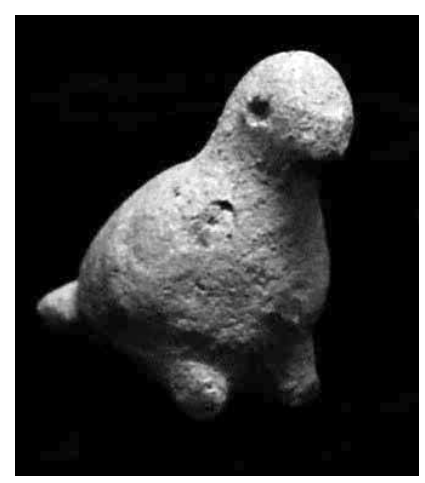

Coordinador del estudio:

Álvaro Bermejo

Etnomusicólogo

Universidad del Atlántico, Colombia.

Ocarina Zenú. Aerófono soplado ornitoforma de la cultura Zenú, del período "formativo medio" con una data de 450 d.C., ubicada en el área geográfica Purísima del Departamento de Córdoba. Construida de cerámica, con dimensiones aproximadas de $5 \times 4 \mathrm{~cm}$.

El instrumento fue encontrado en los procesos de excavaciones arqueológicas de Gerardo Reichell Dolmatoff. Actualmente esta ocarina está ubicada en el Museo de Antropología de la Universidad del Atlántico.

La "ocarina" es un nombre de origen italiano dado a la familia de flautas globulares con conducto de aire. Fue un objeto de amplia distribucion y muy abundante en Mesoamérica y los Andes hasta la región de Arica. La cultura Zenú de Colombia (200-1600 d.C.) confeccionó un tipo de "ocarina" muy estable en el tiempo, que probablemente corresponde a un tipo de sonido con posibilidades musicales muy precisas, pero que no han sido estudiadas hasta ahora. Estos ejemplares son de cerámica y tienen una forma alargada, que la hace muy fácil de tañer, y que supone un comportamiento sonoro cercano a todos los tipos de flauta recta conocidos en los Andes, tales como la kena y el pinkullo, a modo de ejemplo.

\section{ILMENITA SONORA OLMECA}

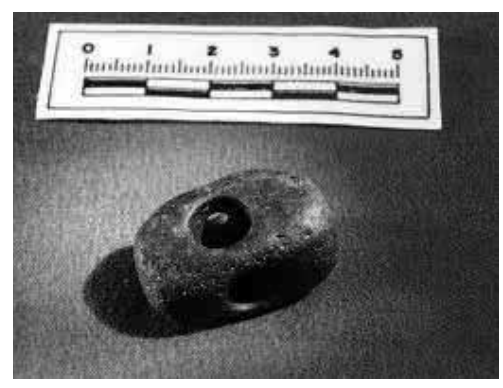

Coordinador del estudio:

Roberto Velázquez

Ingeniero, investigador de aerófonos o resonadores mexicanos

Instituto Virtual de Investigación Tlapitzcalzin, México.

Ilmenita sonora Olmeca. Generador de ruido bucal de roca negra (ilmenita) con tres perforaciones cónicas, sus medidas son: $30 \mathrm{~mm}$ de largo x $19 \mathrm{~mm}$ de ancho y $15 \mathrm{~mm}$ de altura. Fue encontrado casualmente en el despacho del antropólogo Francisco Beverido, ubicada en Xalapa, Veracruz, en el año 1999.

Según el ingeniero Velázquez, la importancia de este objeto sonoro radica en su singular material lítico, tipología y morfología, y porque pudo construirse y usarse hace 3000 años. Produce sonidos que imitan llamados de animales o ruidos del viento y que pueden generar efectos especiales en los humanos.

Su procedencia exacta es desconocida, pero es posible que su origen sea de la zona de influencia Olmeca de San Lorenzo, Veracruz. Su temporalidad es estimativa, posiblemente corresponde a la fase de San Lorenzo, del período "formativo temprano" en 1200-900 a.C.

Este objeto, que se encuentra en el Instituto Virtual de Investigación Tlapizcalzin, se cree que pertenece a la cultura Olmeca (1200-900 a.C.) y que representa una especie organológica única, con características acústicas no compartidas con otros aerófonos. Los experimentos hechos hasta la fecha indican que se comporta como un generador de ruido, es decir, produce un sonido muy especial, poco definible en los términos clásicos de la música occidental. Su particular conformación y las especiales condiciones acústicas que propone su estudio permiten avanzar en el conocimiento de una forma muy particular de la estética sonora regional, que se aleja de lo que se conoce hasta ahora. 


\section{CONCLUSIÓN}

El objetivo específico 4 del proyecto Achalay/CYTED establece que se debe reconstruir, almacenar y conservar en archivos digitales, muestras de sonido del (los) instrumento (s) prehispánico (s) no existente (s) o no accesible (s), seleccionado (s) por la Comisión de Acústica/Instrumentos prehispánicos. Las muestras deben generarse mediante técnicas de modelamiento virtual, a partir de las características acústicas, mecánicas y de ejecución musical resultantes del proceso de reconstrucción arqueomusicológico que ha emprendido la respectiva comisión.

El trabajo de los miembros de la Comisión es modelar y "construir" un instrumento virtual mediante técnicas de modelamiento físico, que genere sonidos de alta calidad y que pueda ser interpretado en tiempo real por músicos profesionales o aficionados en un computador personal de potencia media con el mejor grado de ejecutabilidad.

\section{BIBLIOGRAFÍA}

BARRIENTOS, LINA

2011 Comisión Acústica/Instrumentos Prehispánicos. Informe presentado en la reunión presencial efectuada el 7, 8 y 9 de julio 2011, en dependencias de REUNA, Santiago de Chile. Universidad de La Serena, julio 2011, Chile. Disponible en www.redclara.net/achalai

2012 Informe final Proyecto Achalai/RedCLARA. Presentado en la reunión deRedCLARA/ALICE2 efectuada en Lima-Perú, julio 3 y 4 de 2012. Universidad de La Serena, junio 2012, Chile. Disponible en www.redclara.net/achalai

Bolaños, CÉsar

1988 Las antaras nazca. Lima, Perú: INDEA / CONGyTEC.

1997 "Las antaras y las botellas silbadoras arqueológicas norandinas: una incógnita a dilucidar". MS. 8 pp.

Crespo, Hernán

1966 "Nacimiento y Evolución de la Botella Silbato", Humanitas, VI/1. Quito-Ecuador: Editorial Universitaria, pp. 66-87.

Gerard-Ardenois, Arnaud

1997 "Multifonías en aerófonos andinos de Bolivia", Revista Boliviana de Física, III/3 (agosto). La Paz, Bolivia: Sociedad Boliviana de Física e Instituto de Investigaciones Físicas UMSA, pp. 40-59.

GRUSZCZYNSKa-Ziolkowska, ANNA

1994 "Panpipes Antaras from Nazca (Perú): Problems of Reconstruction”, en Annie Bélis y Catherine Homo-Lechner (editores), La Plurisciplinarité en archéologie musicale: IVe Rencontres Internationales de le Groupe d'Etudes sur l'Archeologie Musicale de l'ICTM, Saint Germain - en - Laye, 8-12 Oct 1990. Volumen II. París: Editions de la maison des sciences de l'homme, pp. 339-346.

Olsen, DALE

1990 "The Ethnomusicology of Archaeology: A Model for the Musical/Cultural Study of Ancient Material Culture". Selected Reports of Ethnomusicology. Volumen 8. Los Angeles: Universidad de California, pp. 175-200. 
Pérez de Arce, José

2011 "Estética sonora prehispánica”. Documento de trabajo. Santiago de Chile, septiembre 2011. Disponible en www.redclara.net/achalai.

1995 "Música prehispánica", en Sonidos de América. Santiago: Museo Chileno de Arte Precolombino y Banco O'Higgins, pp. 19-35.

2000 "Sonido Rajado, Historical Approach", The Galpin Society Journal, N ${ }^{\circ}$ LIII. Londres: The Galpin Society, pp. 233-251.

2004 "Análisis de las cualidades sonoras de las botellas silbadoras prehispánicas de Los Andes", Boletín del Museo Chileno de Arte Precolombino, No 9, pp. 9-34.

REYES, EMIro

2011 Resúmen prospectivo de la reunión de la Comisión de Modelamiento y Tecnología, realizada en Santiago de Chile, entre el 7 y 9 de julio 2011. Universidad de Ibagué, agosto 2011, Colombia. Disponible en www.redclara.net/achalai

VELÁzQuez, RoBERTo

2012 "Cuna con silbato". Publicado en http://www.tlapitzalli.com/, 14 de marzo de 2012, México, 32 pp.

2012 "Arqueociencia sonora". Conferencia incluida en el programa de la $23^{\mathrm{a}}$ Ofrenda del Día Mundial de Nuestra Madre Tierra. Cámara de Diputados, 23 de abril de 2012, México. Publicado en http://www.tlapitzalli.com/, 32 pp.

Otras referencias

http://cienciaysociedad-cyted.ning.com/

http:/ /achalai.redclara.net/

http://www.reuna.cl/index.php/es/comunidades/achalai

www.redclara.net/achalai

www.astraproject.org

http://www.tlapitzalli.com/ 